\title{
CdTe photoelectrochemical solar cells-Chemical modification of surfaces
}

\author{
K C MANDAL, S BASU and D N BOSE \\ Materials Science Centre, Indian Institute of Technology, Kharagpur 721 302, India
}

Abstract. Chemical modification of both $n$ and $p$ type CdTe has been found to improve the performance and stability of PEC solar cells. The surfaces, modified by $\mathrm{Ru}^{3+}$, have been examined by a variety of techniques. Modification results in enhanced barrier height at the surface due to the formation of a passivating oxide layer.

Keywords. Solar cells: cadmium telluride; surface modification.

\section{Introduction}

High efficiency PEC solar cells with GaAs and InP as photoelectrodes have been developed using the technique of chemical modification of surfaces, originating from the work of Parkinson et al (1978) and Heller et al (1980). Tufts et al (1987) recently reported a $15 \%$ efficiency $n$-GaAs PEC cell with $\mathrm{Os}^{3+}$ surface modification which is suitable for a charge transfer $>30,000 \mathrm{C} / \mathrm{cm}^{2}$.

While most of these experiments have been successful on III-V semiconductors, Bose et al (1986) were the first to demonstrate that such surface modification was equally effective on both $n$ and $p$ type CdTe. This paper attempts to summarize the experiments conducted on CdTe surfaces as well as the performance of PEC solar cells. It may be recalled that two alternative mechanisms of chemical modification have been proposed: (i) removal of surface state pinning by Heller (1981) and (ii) electrocatalytic effect by Borbavello et al (1982). While the former results in a decrease of hole recombination at the surface the latter gives rise to increased hole transfer into the electrolyte. The present results show unambiguous evidence of decrease of surface recombination velocity and removal of surface state pinning which is also evident at the $\mathrm{CdTe} /$ air interface. Similar result was earlier reported on $n$-InP electrolyte interfaces (Bose et al 1984a) as well as Schottky barriers (Bose et al 1984b).

\section{Experimental}

The investigations were conducted on Bridgman-grown CdTe which was doped with In (donor) or $\mathrm{P}$ (acceptor) to provide large grain polycrystalline material with the properties as given in table 1.

Table 1. Properties of Bridgman-grown bulk CdTe.

\begin{tabular}{lccc}
\hline Type & $\begin{array}{c}\text { Resistivity } \\
(\text { ohm-cm) }\end{array}$ & $\begin{array}{c}\text { Carrier conc. } \\
\left(\mathrm{cm}^{-3}\right)\end{array}$ & $\begin{array}{c}\text { Mobility } \\
\left(\mathrm{cm}^{2} \mathrm{~V}^{-1} \mathrm{~s}^{-1}\right)\end{array}$ \\
\hline$p$ & 28 & $1.25 \times 10^{16}$ & 16 \\
$n$ & $8 \cdot 7$ & $1.7 \times 10^{15}$ & 428 \\
\hline
\end{tabular}


Surface modification was carried out as described earlier by etching for $30 \mathrm{~s}$ to $1 \mathrm{~min}$ in $8 \mathrm{M} \mathrm{HCl}$ to obtain a black matte surface and then immersing in $0.01 \mathrm{M}$ $\mathrm{RuCl}_{3} 3 \mathrm{H}_{2} \mathrm{O}$ in $0 \cdot 1 \mathrm{M} \mathrm{HNO}_{3}$ for $15 \mathrm{~min}$ which was the optimum period. Comparison was also made with effects of $\mathrm{Pt}^{4+}$ and $\mathrm{Pd}^{2+}$ by immersing in respective chlorides. Unmodified surfaces were prepared by etching in $\mathrm{H}_{2} \mathrm{SO}_{4}: \mathrm{K}_{2} \mathrm{Cr}_{2} \mathrm{O}_{7}$ and finally washed in $2 \%$ bromine-methanol.

\subsection{Results}

$n$ and $p$-CdTe were used as photoelectrodes in $\mathrm{Te}^{2-} / \mathrm{Te}_{2}^{2-}$ and $\mathrm{Sn}^{2+} / \mathrm{Sn}^{4+}$ redox electrolytes respectively in which stable performance has been reported. Details of the experiments have been described elsewhere (Mandal et al 1986, 1987). Apart from dark I-V, C-V and solar cell characteristics, spectral response and $\ln (1-\eta)$ vs $V_{b}^{1 / 2}$ plots were also determined. From the intercepts, the values of diffusion lengths before and after modification were found. The results are summarized in table 2 .

Similar experiments were conducted using $\mathrm{Pt}^{4+}$ and $\mathrm{Pd}^{2+}$ which showed that while the former had some beneficial effect, the latter degraded the cell properties.

\subsection{Surface characterization}

To examine the reasons for surface modification, corroborative studies were conducted on measurement of contact potential difference (CPD) (Bose et al 1986), sub-band gap spectroscopy and electrochemical photocapacitance spectroscopy (EPS) (Bose et al 1988). These experiments proved that (i) negative surface charge developed on $n$ CdTe and positive on $p$-CdTe increasing the barrier height in each case such that $\Delta V_{\mathrm{CPD}} \simeq \Delta V_{\text {oc }}$. (ii) The order of magnitude in sub-band response reduced on modification in both cases. (iii) Discrete levels (surface and bulk) present in CdTe were altered on matte etching and further changed on surface modification. Mid-gap recombination centres disappeared while states which were suitable for minority carrier transport appeared near the band-edges.

The physical properties of the surfaces were examined using XPS, SIMS and Raman spectroscopy (Bose et al 1985; Bose and Holz 1987). These results can be summarized as follows: (i) the unmodified surfaces ( $n$ and $p$ ) had a thin surface layer of $\mathrm{Te}$ on stoichiometric $\mathrm{CdTe}$; (ii) the modified surfaces were covered by a layer of $\mathrm{TeO}_{2 \pm x}$ with depletion of $\mathrm{Cd}$ towards the outer surface; (iii) $\mathrm{Ru}$ was detected on the modified surface but its charge state could not be determined.

Table 2. Comparison of properties of $n$ and $p$-CdTe before and after Ru surface modification.

\begin{tabular}{llccccccccc}
\hline Type & Surface & $\begin{array}{c}L_{p} \\
(\mu \mathrm{m})\end{array}$ & $\begin{array}{c}s \\
(\mathrm{~cm} / \mathrm{s})\end{array}$ & $\begin{array}{c}J_{\mathrm{o}} \\
\left(\mathrm{A} / \mathrm{cm}^{2}\right)\end{array}$ & $n$ & $\begin{array}{c}V_{\mathrm{fb}} \\
(\mathrm{V})\end{array}$ & $\begin{array}{c}J_{\mathrm{sc}} \\
\left(\mathrm{mA} / \mathrm{cm}^{2}\right)\end{array}$ & $\begin{array}{c}V_{\text {oc }} \\
(\mathrm{V})\end{array}$ & $\begin{array}{c}\text { F.F } \\
\text { lity }\end{array}$ \\
\hline$n$ & Unmod. & 0.12 & $4 \times 10^{4}$ & $8.6 \times 10^{-8}$ & 2.12 & -0.97 & 3.4 & 0.52 & 0.42 & \\
& Mod. & 0.17 & $8.6 \times 10^{3}$ & $4.2 \times 10^{-9}$ & 1.16 & -1.20 & 5.2 & 0.78 & 0.51 & \\
& Unmod. & 0.72 & $1.8 \times 10^{5}$ & $2.8 \times 10^{-7}$ & 2.72 & +1.02 & 2.17 & 0.63 & 0.38 & $18 \mathrm{~h}$ \\
& Mod. & 0.99 & $3.5 \times 10^{4}$ & $5.1 \times 10^{-8}$ & 1.54 & +1.20 & 3.23 & 0.92 & 0.48 & $336 \mathrm{~h}$ \\
\hline
\end{tabular}




\section{Discussion}

The above results can be explained on the basis of Heller's (1981) theory of removal of surface state pinning due to strong chemisorption of $\mathrm{Ru}^{3+}$, since reduction of active surface state density within the band-gap has been directly demonstrated. Further, the grain boundary passivation leading to increase in $J_{\mathrm{sc}}$ also occurs. The interaction of $\mathrm{Ru}$ with $\mathrm{CdTe}$ surface has also been shown in the solid-state Schottky barrier in which increase in $\phi_{b}$ and decrease in ideality factor $n$ has been found (Mandal et al 1988). This is in contrast with the observation of Tufts et al (1987). However improved minority carrier transport to redox electrolyte may also be occurring due to creation of favourable energy states.

The efficacy of $\mathrm{Ru}$ treatment in the present case is considered to be due to the following factors: (a) variable valency of $\mathrm{Ru}$ which allows amphoteric behaviour, (b) smallest atomic radius in its family permitting strong surface interaction and (c) strongly oxidizing nature vis-a-vis $\mathrm{Pt}$ and $\mathrm{Pd}$, the latter being known to be a hydrogenation catalyst. Surface modification by appropriate ions is thus a powerful tool for improving the properties of semiconductor surfaces and interfaces and may find application in diverse fields.

\section{References}

Borbavello E, Kalyansundaran K, Graetzel M and Pelezetti E 1982 Helv. Chim. Acta 65243

Bose D N, Ramprakash Y and Basu S 1984a J. Electrochem. Soc. 131850

Bose D N, Roy J N and Basu S 1984b Mat. Lett. 2455

Bose D N, Hegde M S, Basu S and Mandal K C 1985 Proc. 18th IEEE Photovoltaic Specialists Conf. (New York: IEEE Press)

Bose D N, Basu S, Mandal K C and Mazumdar D 1986 Appl. Phys. Lett. 46472

Bose D N and Holz M 1987 Mat. Lett. 5291

Bose D N, Basu S and Mandal K C 1988 Proc. 7th Int. Conf. on Thin Films, New Delhi

Heller A $1981 \mathrm{Am}$. Chem. Soc. Symp. Ser. 14657

Heller A, Miller B, Lewerenz H J and Bachmann K J 1980 J. Am. Chem. Soc. 1026555

Mandal K C, Basu S and Bose D N 1986 Solar Cells 1825

Mandal K C, Basu S and Bose D N 1987 J. Phys. Chem. 914011

Mandal K C, Basu S and Bose D N 1988 (to be published)

Parkinson B A, Heller A and Miller B 1978 Appl. Phys. Lett. 33521

Tufts B J, Abrahams I L, Santangelo P S, Ryba G N, Casagrande L G and Lewis N S 1987 Nature (London) 326861 